\title{
Editorial
}

\section{Anaesthetic care and audit: 150 years on}

This year marks the 150th anniversary of the first public demonstration of anaesthesia. This is an event which stimulates reflection on how much medicine and surgery have been advanced by various aspects of anaesthetic care. This event is also cause for celebration when we consider how much suffering has been alleviated. Twelve years after this event, an anaesthetist was able to state: "Besides the great benefit conferred by chloroform in the prevention of pain, it probably confers still greater advantages by the extension which it gives to the practice of surgery. Many operations take place... which could not be performed in the waking state.... The surgeon also obtains the ready assent of his patient to a number of other operations, where it would either not be obtained at all, or not at the most favourable time, if the patient had to suffer the pain of them".

In addition, the anniversary also marks the start of efforts by a group of medical practitioners to evaluate the care they provide, in the hope of future improvement. These efforts began with John Snow, the 'father' of epidemiology in anaesthesia. Snow made careful records of his anaesthetics and reviewed his own and others' cases, attempting to draw conclusions about the cause of death. For example, he reviewed in detail fifty "fatal cases of inhalation of chloroform," as well as some eight "alleged fatal cases." In his analysis, Snow discounted "idiosyncracy," "sitting posture," and "effect of surgeon's knife," to name but three factors.

It is in this tradition of observation and analysis of anaesthetic care that Rose and colleagues have examined the risk of postoperative admission to a critical care unit (CCU). In this study, they examined the "incidence, reasons, and timing of critical care admissions for postoperative ventilation within 48 hours of noncardiac and non-neurosurgical procedures at a tertiary-care hospital". 2 Information was collected prospectively over a period of 27 months on 15,059 patients from three separate sources: anaesthetic, critical care and hospital databases. These provided age, ASA physical status classifi-

From the Department of Anaesthesia, University of Calgary, Foothills Hospital, Calgary, Alberta. cation, preoperative illnesses, duration of mechanical ventilation / CCU stay / hospital stay and in-hospital mortality. Patients were not included in the study if they were admitted to the $\mathrm{CCU}$ preoperatively, daycare patients, undergoing cardiac or neurosurgical procedures, or admitted to the CCU but without undergoing mechanical ventilation. The authors classified the patients as to whether or not the admission was planned (P) preoperatively, unplanned (U-1) but with a problem recognised in the operating room (OR) or postanaesthetic recovery unit (PACU), or unplanned (U-2) with a problem recognised within 48 hours postoperatively and after discharge from the PACU. The rate of CCU admission was determined and the characteristics of those admitted were compared to those who were not.

\section{What did they find?}

First, there were certain preoperative patient characteristics or risk factors which were "markers" for CCU admission, in comparison to those patients who were not admitted to the CCU. The preoperative condition of the patient determined a planned admission - more likely to be elderly, male, smokers, and with an ASA classification of 4-5. (The latter reflected the presence of chronic obstructive lung disease, cardiac disease or renal disease.) An unplanned admission from the ward was more likely if the patients were HIV positive. The preoperative physiological feature common to all three groups was that of hemoglobin saturation $<90 \%$. The surgical procedure was also a determinant: - emergency or major abdominal surgery for both P and U-1 and bronchoscopy for U-2.

Second, there were intraoperative markers. Patients in all three groups were more likely to experience tachycardia and haemoglobin desaturation. Group P patients were characterised as experiencing hypotension, dysrhythmia, and increased requirement for packed red blood cells. (These characteristics could not be said to "increase the risk of a planned admission," as stated by the authors, as the decision had been made to admit the patient to the CCU preoperatively.) Group U-2 patients were more likely to have received a "neurolept analgesic technique." 
Third, postoperative admission to the $\mathrm{CCU}$ was described by the authors as an "uncommon" event, as the cases represented only $2.2 \%$ of inpatient procedures, and only $12 \%$ of admissions were not planned. Fourth, when it did occur postoperatively, CCU admission was prompted in $90 \%$ of the cases by either respiratory and/or cardiac complications, with neurological problems being rare.

\section{What can anaesthetists learn from this study?}

The single characteristic which marked admissions to the CCU was that of preoperative haemoglobin saturation of less than $90 \%$ either preoperatively and/or intraoperatively. The lesson for anaesthetists may be the potential usefulness of incorporating oxygen saturation into the normal preoperative regime of measurement of vital signs. Thus, as well as heart rate, blood pressure, respiratory rate, and (occasionally) temperature, a patient's peripheral arterial oxygen saturation can be easily measured and recorded. This measurement could then be incorporated into the process of preoperative evaluation, one of the aims of which is to be able to predict and influence postoperative outcome. However, this assumes validity of preoperative haemoglobin desaturation as causal predictor of poor outcome, not just related to admission to a CCU. Detailed investigation is obviously required.

In addition, Canadian anaesthetists, particularly those engaged in quality assurance activities, now have another set of results with which they may be able to compare their own practice. Or can they? Rose et al. also state that "over $40 \%$ of critical care admissions are for postoperative patients." Unfortunately they do not provide us with their percentage, but cite data from the United States. Data for a similar period from the CCU at Foothills Hospital showed that one-third of admissions were for postoperative patients. ${ }^{3}$ However, this illustrates one of the points made by Rose $e t$ al., that it is very difficult to compare the results from different studies, in part because of the variation in definitions used. For example, the Foothills Hospital data are for all postoperative admissions, including some cardiac surgery and neurosurgery. Another difficulty arises when different study periods are used, even by the same researchers. For example, a previous study by the same authors examined the "frequency of acute, unanticipated respiratory problems" in the PACU. ${ }^{4}$ That study period was for 39 months and overlapped that of the current work. Readers who might wish to analyse the results of the two papers together are therefore not able to do so.

What does this study tell us?

Using three large database systems, Rose et al. have been able to determine that patients with certain characteristics are more likely to be admitted to the CCU than patients who do not share these characteristics. However, the authors have not been able to tell us much about the outcome of these patients, with the exception of duration of ventilation, CCU and hospital stay, and in-hospital mortality rate. Of these, only in-hospital mortality for patients in group U-2 was significantly different from patients in group P. Nor can Rose et al. state if the outcome of planned admission to the CCU was beneficial.

This type of study represents a considerable investment in terms of both finances and hours spent. For example, all charts $(n=329)$ in the two unplanned groups were reviewed by one of the authors. Yet are we any further ahead than was John Snow, who was able to determine that a death during the administration of ether was not due to any intrinsic toxic properties of the anaesthetic agent, but to "want of admission of sufficient air to the lungs"?' Rose et al. have given us some answers as to 'what' can occur to patients with certain characteristics. They admit that the "reasons for unanticipated critical care admission are multifactorial" and that "many may be beyond the scope of the anaesthetist's role in patient care." However, should not anaesthetists use this anniversary as their own marker of new efforts to understand the 'why' of postoperative outcome?

\section{L'audit et l'anesthésie: déjà 150 ans}

Cette année marque le cent cinquantième anniversaire de l'anesthésie. C'est un bon moment pour se rappeler à quel point l'anesthésie a fait progresser la médecine et la chirurgie. On ne peut s'empêcher de célébrer en pensant à la souffrance qui a été soulagée. Douze ans après cette grande découverte, un anesthésiste déclarait: « Tout en procurant des bienfaits immenses en prévenant la douleur, le chloroforme contribue d'avantage au progrès de la chirurgie ... beaucoup d'interventions irréalisables à l'état vigile ... peuvent maintenant être réalisées. Aujourd'hui, le chirurgien obtient le consentement du patient pour bon nombre d'opérations additionnelles, ce 
qu'il ne pouvait obtenir auparavant ou au moment propice, alors que le patient devait en supporter toutes les douleurs $» .^{1}$

Cet anniversaire souligne aussi le début des démarches d'un groupe de praticiens qui voulaient évaluer les soins qu'ils prodiguaient avec l'espoir de les améliorer ultérieurement. Ce travail commence avec John Snow, le père de l'épidémiologie en anesthésie. Snow compilait les compte rendu de ses anesthésies et les analysait ainsi que ceux de ses collègues dans le but de tirer des conclusions sur les causes de décès. Par exemple, il a révisé en détails " quarante cas fatals d'inhalation de chloroforme » de même que « huit cas présumés fatals ». Pendant cette analyse, Snow a écarté « l'idiosyncrasie », « la position assise » et « l'effet du bistouri $»$ pour ne nommër que ces trois facteurs.

C'est dans cette tradition d'observation et d'analyse appliquées à l'anesthésie que Rose et al. ont étudié l'admission postopératoire à l'unité de soins intensifs (USI). Ils ont examiné « dans un centre hospitalier de soins tertiaires, l'incidence, les raisons et le moment de l'admission pour la ventilation postopératoire, en deçà de 48 heures de l'intervention et en excluant la chirurgie cardiaque et neurologique $"{ }^{2}$ Les données de 15059 patients ont été recueillies de façon prospective sur une période de 27 mois à partir des banques de données de l'anesthésie, des soins intensifs et de l'hôpital. Ces banques donnaient accès à l'âge, l'état physique ASA, les maladies préopératoires, la durée de la ventilation/séjour hospitalier et la mortalité intrahospitalière. Ont été exclus, les patients admis à l'USI avant l'intervention, les patients ambulatoires, les opérés cardiaques et neurochirurgicaux et les patients admis à l'USI dont la condition n'exigeait pas de ventilation mécanique. Les auteurs ont classifié les admissions selon qu'elles étaient planifiées $(P)$ ou non en préopératoire, non planifiées (U-1) mais avec un problème survenu en salle d'opération) ou à l'unité des soins postanesthésique, ou non planifiées (U-2) avec un problème découvert dans les 48 heures postopératoires et après le congé de l'unité de soins postanesthésiques. Le taux d'admission à l'USI a été déterminé et les caractéristiques de ceux qui étaient admis comparées à celles de ceux qui ne l'étaient pas.

\section{Qu'ont-ils trouvé?}

D'abord, ils ont noté certaines caractéristiques ou facteurs préopératoires qui étaient des « marqueurs » d'admission à l'USI, qui n'existaient pas chez ceux qui n'étaient pas admis. La condition préopératoire du patient déterminait l'admission planifiée - plus vraisemblablement l'âge avancé, le tabagisme et la classification 4-5 de 1'ASA (celle-ci étant le reflet d'une maladie obstructive chronique, d'une cardiopathie ou d'une néphro- pathie). La cause la probable de l'admission non planifiée était la séropositivité HIV. Le dénominateur physiologique préopératoire commun aux trois groupes était la désaturation $<90 \%$. L'intervention chirurgicale était aussi déterminante: - la chirurgie urgente ou abdominale majeure pour les classes $\mathrm{P}$ et $\mathrm{U}-1$ et la bronchoscopie pour la classe U-2.

Deuxièmement, ils ont aussi trouvé des « marqueurs » peropératoires. Les patients des trois groupes souffraient plus souvent de tachycardie et de désaturation en oxygène. Le groupe $P$ était caractérisé par l'hypotension, la dysrythmie et l'utilisation accrue d'hématies concentrées (on ne peut dire que ces caractéristiques augmentaient la possibilité d'une admission planifiée comme le prétendent les auteurs, parce que la décision d'admettre le patient à l'USI avait déjà été prise avant l'intervention). Les patients du groupe U-2 avaient le plus souvent reçu une neuroleptanalgésie.

Troisièmement, les auteurs considéraient l'admission postopératoire à l'USI comme un événement rare, n'étant survenu que pour $2,2 \%$ des interventions chez les patients hospitalisés, alors que seulement $12 \%$ des admissions à l'USI n'étaient pas planifiées. Quatrièmement, quand elle était nécessaire à la période postopératoire, l'admission à l'USI était occasionnée dans $95 \%$ des cas par des complications respiratoires et/ou cardiaques, rarement par des complications neurologiques.

Que l'anesthésiste peut-il apprendre de cette étude? Une désaturation artérielle en oxygène inférieure à $90 \%$, que ce soit avant ou pendant l'intervention, représentait la caractéristique la plus commune toutes les admissions à l'USI. Les anesthésistes peuvent donc conclure qu'il serait utile d'inclure la mesure préopératoire de la saturation dans le répertoire des signes vitaux. En effet, comme la fréquence cardiaque, la pression artérielle, la fréquence respiratoire et (à l'occasion) la température, la saturation artérielle périphérique en oxygène se mesure et s'enregistre facilement. Cette mesure pourrait être intégrée au processus de l'évaluation préopératoire dans le but de prédire et d'influencer le pronostic. Cependant, on assume ainsi que la désaturation en préopératoire de l'hémoglobine est une cause validée d'un résultat défavorable, non seulement une cause d'admission à l'USI. De toute évidence, une investigation en profondeur s'avère nécessaire.

De plus, les anesthésistes canadiens, surtout ceux qui se sont engagés dans le processus d'assurance-qualité, possèdent maintenant un autre profil de résultats avec lesquels ils peuvent comparer leur propre pratique. Ou peuvent-ils? Rose et al. mentionnent aussi que $40 \%$ des àdmissions à l'USI surviennent en postopératoire. 
Malheureusement, ils ne proposent pas leur propre pourcentage, mais ne citent que des données provenant des Etats-Unis. Pendant une période sembable, les données recueillies à l'USI du Foothills Hospital ont révélé que le tiers des admissions se situait à la période postopératoire. ${ }^{3}$ Cependant, ceci illustre un des point soulevés par Rose et al:: il est très difficile de comparer les résultats provenant de plusieurs études, en partie parce que les définitions diffèrent. Par exemple, les données du Foothills Hospital se rapportent à toutes les admissions postopératoires incluant la chirurgie cardiaque et la neurochirurgie. Une autre difficulté survient lorsque les périodes d'études sont différentes, même si les études sont réalisées par le même chercheur. Par exemple, une étude antérieure des mêmes auteurs portait sur la fréquence des problèmes respiratoires aigus non anticipés à l'unité des soins postopératoires. ${ }^{4}$ La période d'étude était de 39 mois et empiétait sur celle du travail actuel. Par conséquent, il est impossible aux lecteurs qui le désirent d'analyser les résultats des deux travaux.

\section{Qu'est-ce que cette étude nous dit?}

A l'aide de trois systèmes considérables de bases de données, Rose et al. ont été capables de déterminer que les patients qui possèdent certaines caractéristiques sont plus sujets à être admis à l'USI que les patients qui ne partagent pas ces caractéristiques. Cependant, les auteurs ont été incapables de nous en dire beaucoup sur le devenir de ces patients, à l'exception de la durée de la ventilation, de la durée du séjour à l'ISU et à l'hôpital, et le taux de mortalité hospitalière. De ces résultats, seul le taux de mortalité hospitalière des patients du groupe U-2 était significativement différent de celui des patients du groupe P. Rose et al. ne peuvent non plus conclure que l'admission à l'ISU était bénéfique.

Ce type d'étude représente un investissement considérable en temps et en argent. Par exemple, tous les dossiers $(n=329)$ du groupe non planifié ont été révisés par un de auteurs. Jusqu'à maintenant, avons-nous réellement pris de l'avance sur John Snow, qui a été capable de déterminer que la mort survenant pendant l'anesthésie à l'éther n'était pas due à une propriété intrinsèque de l'argent anesthésique, " mais a un défaillance de l'arrivée d'air aux poumons $»$ ? $^{1}$ Rose $e t$ al. nous ont donné quelques réponse sur ce qui peut arriver à des patients qui possèdent certaines caractéristiques. Ils admettent que les causes d'admissions non anticipées aux soins intensifs sont multifactorielles et que plusieurs peuvent outrepasser la mission de l'anesthésiste. Cependant, les anesthésistes ne devraient-ils pas utiliser cet anniversaire comme le point de départ de leurs nouveaux efforts à la recherche du pourquoi des résultats postopératoires?

\section{References}

1 Snow J. On Chloroform and Other Anaesthetics: Their Action and Administration. London: John Churchill, 1858.

2 Rose DK, Byrick RJ, Cohen MM, Caskennette GM.

Planned and unplanned postoperative admissions to critical care for mechanical ventilation. Can J Anaesth 1996; 43: 333-40.

3 Rose DK, Cohen MM, Wigglesworth DF, DeBoer DP. Critical respiratory events in the postanesthesia care unit. Anesthesiology 1994; 81: 410-8.

4 Sandham D. Personal communication, 1996. 